\title{
STRESS DISTRIBUTION AROUND A RIGID LINE IN DISSIMILAR MEDIA
}

\author{
C. P. JIANG and C. T. LIU \\ Institute of Mechanics, Chinese Academy of Sciences, Beijing 100080, P.R.C.
}

\begin{abstract}
The elastic plane problem of a rigid line inclusion between two dissimilar media was considered. By solving the Riemann-Hilbert problem, the closed-form solution was obtained and the stress distribution around the rigid line was investigated. It was found that the modulus of the singular behavior of the stress remains proportional to the inverse square root of the distance from the rigid line end, but the stresses possess a pronounced oscillatory character as in the case of an interfacial crack tip.
\end{abstract}

\section{INTRODUCTION}

THE INTERFACIAL fracture problem has aroused great interest. The presence of inclusions at interfaces plays an important role in the fracture behavior. For the elastic plane problem of bonded dissimilar media, if the value of the elastic modulus of a flat inclusion is much greater than that of every matrix, it appears reasonable to consider it as a rigid line. From the viewpoint of inhomogeneities in solids, a rigid line and a slit crack are the two extreme cases of a flat inhomogeneity, namely, for a rigid line $E \rightarrow \infty$, and for a crack $E \rightarrow 0$, where $E$ is Young's modulus. For structural integrity assessments, these are the two extreme cases which result in stress concentrations and may result in rupture.

Hitherto, a number of investigations on rigid lines in the homogeneous plane have been conducted. In ref. [1], Muskhelishvili gave a solution for a rigid line under a uniform stress state at infinity. Works by Li and Ting [2], Hao and Wu [3], Markenscoff and Dundus [4], and Jiang [5] provide examples of recent contributions.

As a logical extension of the previous works, in this paper an attempt was made to find the solution to the elastic plane problem of a rigid line between two bonded dissimilar media, and to investigate the stress distribution around a rigid line end.

\section{STATEMENT OF THE PROBLEM}

The problem to be considered is as follows. Referring to Fig. 1, let medium I with elastic constants $\mu_{1}, \kappa_{1}$ and medium II with $\mu_{2}, \kappa_{2}$ occupy the upper and lower half-planes, $S^{+}$and $S^{-}$, respectively, where $\mu_{j}$ is the shear modulus, and $\kappa_{j}=3-4 v_{j}$ for plane strain, $\kappa_{j}=\left(3-v_{j}\right) /\left(1+v_{j}\right)$ for generalized plane stress, $v_{j}$ being Poisson's ratio. Let a rigid line lie along a part, $L$, of the real axis which is the bond line of the two media. Let $L^{\prime}$ be the remainder of the real axis. Then the boundary conditions of stresses $\left(\sigma_{x}, \sigma_{y}, \tau_{x y}\right)$ and displacements $(u, v)$ for the problem may be expressed as follows:

$$
\begin{gathered}
\left(\sigma_{y 1}-i \tau_{x y 1}\right)^{+}=\left(\sigma_{y 2}-i \tau_{x y 2}\right)^{-} \quad \text { on } L^{\prime} \\
\left(u_{1}+i v_{1}\right)^{+}=\left(u_{2}+i v_{2}\right)^{-} \text {on } L^{\prime} \\
\left(u_{1}+i v_{1}\right)^{+}=u_{0}+i v_{0}+i \delta x \text { on } L \\
\left(u_{2}+i v_{2}\right)^{-}=u_{0}+i v_{0}+i \delta x \text { on } L
\end{gathered}
$$

where subscripts 1 and 2 refer to media I and II, respectively, superscripts + and - refer to the value of the functions on the real axis as approached from $S^{+}$and $S^{-}$, respectively, $u_{0}$ and $v_{0}$ are the displacements of the midpoint of the rigid line and $\delta$ is the rotation of the rigid line. Taking 


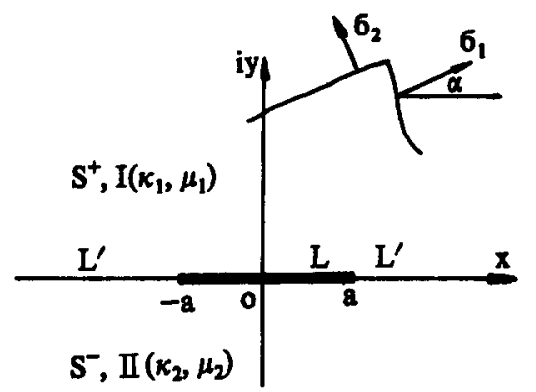

Fig. 1. A rigid line between two bonded dissimilar half-planes.

the derivative of (2.3) and (2.4), with respect to $x$, the addition and subtraction of these two equations yield:

$$
\begin{gathered}
\left(u_{1}^{\prime}+i v_{1}^{\prime}\right)+\left(u_{2}^{\prime}+i v_{2}^{\prime}\right)^{-}=2 i \delta \text { on } L \\
\left(u_{1}^{\prime}+i v_{1}^{\prime}\right)^{+}-\left(u_{2}^{\prime}+i v_{2}^{\prime}\right)^{-}=0 \text { on } L
\end{gathered}
$$

where

$$
u_{j}^{\prime}=\frac{\partial u_{j}}{\partial x}, \quad v_{j}^{\prime}=\frac{\partial v_{j}}{\partial x}
$$

In addition, to determine solely the solution to the problem, the equilibrium conditions of the rigid line must be considered. Assuming that the rigid line is traction-free, we have

$$
\begin{array}{r}
\int_{-a}^{a}\left(\sigma_{y 1}-i \tau_{x y 1}\right)^{+} \mathrm{d} x-\int_{-a}^{a}\left(\sigma_{y 2}-i \tau_{x y 2}\right)^{-} \mathrm{d} x=0 \\
\int_{-a}^{a} x \sigma_{y 1}^{+} \mathrm{d} x-\int_{-a}^{a} x \sigma_{y 2}^{-} \mathrm{d} x=0 .
\end{array}
$$

Apparently, (2.9) can be rewritten as

$$
\operatorname{Re} \int_{-a}^{a} x\left(\sigma_{y 1}-i \tau_{x y 1}\right)^{+} \mathrm{d} x-\operatorname{Re} \int_{-a}^{a} x\left(\sigma_{y 2}-i \tau_{x y 2}\right)^{-} \mathrm{d} x=0
$$

\section{FORMULATION OF THE PROBLEM AND ITS SOLUTION}

To formulate the problem, we use the complex stress functions $\Phi_{1}(z)$ and $\Psi_{1}(z)$ which refer to medium I and are defined in $S^{+}[1]$. Applying the Riemann-Schwarz symmetry principle, another analytic function $\Omega_{1}(z)$, which is defined in $S^{-}$, can be introduced:

$$
\Omega_{1}(z)=\Phi_{1}(z)+z \Phi_{1}^{\prime}(z)+\Phi_{1}(z) \quad z \in S^{-}
$$

where $\Phi_{1}(z)=\overline{\Phi_{1}(\bar{z})}$. For an arbitrary uniform stress state at infinity, we have

$$
\begin{aligned}
& \Phi_{1}(z)=\Gamma_{1}+\Phi_{10}(z) \quad z \in S^{+} \\
& \Omega_{1}(z)=\Gamma_{1}+\Gamma_{1}^{\prime}+\Omega_{10}(z) \quad z \in S^{-}
\end{aligned}
$$

where $\Phi_{10}(z)$ and $\Omega_{10}(z)$ are holomorphic in $S^{+}$and $S^{-}$, respectively, vanish at infinity, and

$$
\Gamma_{1}=\frac{1}{4}\left(\sigma_{1}+\sigma_{2}\right)+i \frac{2 \mu_{1}}{1+\kappa_{1}} \varepsilon_{1}^{\infty} \quad \Gamma_{1}^{\prime}=-\frac{1}{2}\left(\sigma_{1}-\sigma_{2}\right) \mathrm{e}^{-2 k \alpha}
$$

where $\sigma_{1}$ and $\sigma_{2}$ are the principal stresses at infinity in the upper half-plane, $\varepsilon_{1}^{\infty}$ is the rotation at infinity and $\alpha$ is the angle between $\sigma_{1}$ and the $O x$ axis.

Similarly, we can use $\Phi_{2}(z)$ and $\Omega_{2}(z)$, which refer to medium II and for which

$$
\begin{aligned}
& \Phi_{2}(z)=\Gamma_{2}+\Phi_{20}(z) \quad z \in S^{-} \\
& \Omega_{2}(z)=\Gamma_{2}+\Gamma_{2}^{\prime}+\Omega_{20}(z) \quad z \in S^{+}
\end{aligned}
$$

where $\Phi_{20}(z)$ and $\Omega_{20}(z)$ are holomorphic in $S^{-}$and $S^{+}$, respectively, and vanish at infinity. 
The basic equations for two-dimensional classical elasticity in the form used by Muskhelishvili are

$$
\begin{aligned}
\sigma_{x j}+\sigma_{y j} & =2\left[\Phi_{j}(z)+\overline{\Phi_{j}(z)}\right] \\
\sigma_{y j}-i \tau_{x y j} & =\Phi_{j}(z)+\Omega_{j}(\bar{z})+(z-\bar{z}) \overline{\Phi_{j}^{\prime}(z)} \\
2 \mu_{j}\left(u_{j}^{\prime}+i v_{j}^{\prime}\right) & =\kappa_{j} \Phi_{j}(z)-\Omega_{j}(\bar{z})-(z-\bar{z}) \overline{\Phi_{j}^{\prime}(z)} .
\end{aligned}
$$

From (2.2) and (2.6), we have

$$
\left(u_{1}^{\prime}+i v_{1}^{\prime}\right)^{+}=\left(u_{2}^{\prime}+i v_{2}^{\prime}\right)^{-} \text {on } L+L^{\prime}
$$

Substituting (3.9) into (3.10), we obtain

$$
\frac{\kappa_{1}}{\mu_{1}} \Phi_{1}^{+}(t)-\frac{1}{\mu_{1}} \Omega_{1}^{-}(t)=\frac{\kappa_{2}}{\mu_{2}} \Phi_{2}^{-}(t)-\frac{1}{\mu_{2}} \Omega_{2}^{+}(t) \text { on } L+L^{\prime}
$$

where $t$ denotes the coordinate on the real axis.

Substituting (3.2), (3.3), (3.5), (3.6) into (3.11) and arranging, it is seen that

$$
\begin{aligned}
{\left[\frac{\kappa_{1}}{\mu_{1}} \Phi_{10}(t)+\frac{1}{\mu_{2}} \Omega_{20}(t)\right]^{+}=\left[\frac{\kappa_{2}}{\mu_{2}} \Phi_{20}(t)\right.} & \left.+\frac{1}{\mu_{1}} \Omega_{10}(t)\right]^{-}-\frac{\kappa_{1}}{\mu_{1}} \Gamma_{1} \\
& -\frac{1}{\mu_{2}}\left(\Gamma_{2}+\Gamma_{2}^{\prime}\right)+\frac{\kappa_{2}}{\mu_{2}} \Gamma_{2}+\frac{1}{\mu_{1}}\left(\Gamma_{1}+\Gamma_{1}^{\prime}\right) \text { on } L+L^{\prime} .
\end{aligned}
$$

According to Liouville's theorem and noting the behavior of $\Phi_{j 0}(z)$ and $\Omega_{j 0}(z)$ at infinity, we obtain

$$
\begin{aligned}
\frac{\kappa_{1}}{\mu_{1}} \Phi_{10}(z)+\frac{1}{\mu_{2}} \Omega_{20}(z)=0 \quad z \in S^{+} & \frac{\kappa_{2}}{\mu_{2}} \Phi_{20}(z)+\frac{1}{\mu_{1}} \Omega_{10}(z)=0 \quad z \in S^{-} \\
-\frac{\kappa_{1}}{\mu_{1}} \Gamma_{1}-\frac{1}{\mu_{2}}\left(\Gamma_{2}+\Gamma_{2}^{\prime}\right)+\frac{\kappa_{2}}{\mu_{2}} \Gamma_{2}+\frac{1}{\mu_{1}}\left(\Gamma_{1}+\Gamma_{1}^{\prime}\right)=0 . &
\end{aligned}
$$

The substitution of (3.8) into (2.1) yields

$$
\left[\Phi_{10}(t)-\Omega_{20}(t)\right]^{+}=\left[\Phi_{20}(t)-\Omega_{10}(t)\right]^{-}-\Gamma_{1}+\Gamma_{2}+\Gamma_{2}^{\prime}+\Gamma_{2}-\Gamma_{1}-\Gamma_{1}^{\prime} .
$$

Let

$$
\theta(z)= \begin{cases}\Phi_{10}(z)-\Omega_{20}(z) & z \in S^{+} \\ \Phi_{20}(z)-\Omega_{10}(z) & z \in S^{-}\end{cases}
$$

Then it is seen that $\theta(z)$ is holomorphic in the whole plane cut along $L$ and vanishes at infinity, and

$$
\Gamma_{1}-\Gamma_{2}-\Gamma_{2}^{\prime}=\Gamma_{2}-\Gamma_{1}-\Gamma_{1}^{\prime}
$$

Equations (3.15) and (3.18) show that if $\Gamma_{1}$ and $\Gamma_{1}^{\prime}$ are prescribed, $\Gamma_{2}$ and $\Gamma_{2}^{\prime}$ will be determined. As an example, assuming that

$$
\sigma_{x 1}^{\infty}=0, \quad \sigma_{y 1}^{\infty}=\sigma^{\infty}, \quad \tau_{x y !}^{\infty}=0, \quad \varepsilon_{1}^{\infty}=0
$$

then

$$
\Gamma_{1}=\frac{1}{4} \sigma^{\infty}, \quad \Gamma_{1}^{\prime}=\frac{1}{2} \sigma^{\infty}
$$

From (3.15) and (3.18), we obtain:

$$
\Gamma_{2}=\frac{\mu_{2}\left(\kappa_{1}-3\right)+4 \mu_{1}}{4 \mu_{1}\left(\kappa_{2}+1\right)} \sigma^{\infty}, \quad \Gamma_{2}^{\prime}=\frac{2 \mu_{1}\left(\kappa_{2}-1\right)-\mu_{2}\left(\kappa_{1}-3\right)}{2 \mu_{1}\left(\kappa_{2}+1\right)} \sigma^{\infty} .
$$


It is seen that $\sigma_{y 2}^{\infty}=\sigma^{\infty}, \tau_{x y 2}^{\infty}=0$, but $\sigma_{x 2}^{\infty} \neq 0$. The stress component $\sigma_{x}$ has a jump across the bond line. In addition, it should be pointed out that the rotations $\varepsilon_{1}^{\infty}$ and $\varepsilon_{2}^{\infty}$ at infinity cannot be set arbitrarily to zero at the same time unless $\tau_{x y j}^{\infty}=0$.

From (3.13), (3.14) and (3.17), we obtain:

$$
\begin{aligned}
& \Phi_{10}(z)=\frac{\mu_{1}}{\kappa_{1} \mu_{2}+\mu_{1}} \theta(z) \quad z \in S^{+} \\
& \Omega_{20}(z)=-\frac{\kappa_{1} \mu_{2}}{\kappa_{1} \mu_{2}+\mu_{1}} \theta(z) \quad z \in S^{+} \\
& \Phi_{20}(z)=\frac{\mu_{2}}{\kappa_{2} \mu_{1}+\mu_{2}} \theta(z) \quad z \in S^{-} \\
& \Omega_{10}(z)=\frac{\kappa_{2} \mu_{1}}{\kappa_{2} \mu_{1}+\mu_{2}} \theta(z) \quad z \in S^{-} .
\end{aligned}
$$

Substituting (3.9) into (2.5), and noting (3.22)-(3.25), we obtain

$$
\theta^{+}(t)-g \theta^{-}(t)=f
$$

where

$$
\begin{aligned}
& g=\frac{\kappa_{2}\left(\kappa_{1} \mu_{2}+\mu_{1}\right)}{\kappa_{1}\left(\kappa_{2} \mu_{1}+\mu_{2}\right)} \\
& f=\frac{\kappa_{1} \mu_{2}+\mu_{1}}{\kappa_{1}}\left[-\left(\frac{\kappa_{1}}{2 \mu_{1}} \Gamma_{1}+\frac{\kappa_{2}}{2 \mu_{2}} \Gamma_{2}\right)+\frac{1}{2 \mu_{1}}\left(\Gamma_{1}+\Gamma_{1}^{\prime}\right)+\frac{1}{2 \mu_{2}}\left(\Gamma_{2}+\Gamma_{2}^{\prime}\right)\right]+2 i \delta
\end{aligned}
$$

The general solution of $(3.26)$ is [1]

$$
\theta(z)=\frac{f}{2 \pi i} X(z) \int_{L} \frac{1}{X^{+}(t)} \cdot \frac{\mathrm{d} t}{t-z}+C X(z)
$$

where $C$ is a complex constant, and

$$
X(z)=(z+a)^{-1 / 2+i \beta}(z-a)^{-1 / 2-i \beta}
$$

which is the single-valued branch, such that $z X(z) \rightarrow 1$ as $|z| \rightarrow \infty$, and in which

$$
\beta=\frac{1}{2 \pi} \ln |g| \text {. }
$$

Noting that

$$
\begin{cases}X^{+}(t)=g X^{-}(t) & \text { on } L \\ X^{+}(t)=X^{-}(t) & \text { on } L^{\prime}\end{cases}
$$

we have

$$
\frac{1}{2 \pi i} \int_{L} \frac{1}{X^{+}(t)} \cdot \frac{\mathrm{d} t}{t-z}=\frac{1}{1-g} \cdot \frac{1}{2 \pi i} \int_{\wedge} \frac{1}{X(\zeta)} \cdot \frac{\mathrm{d} \zeta}{\zeta-z}
$$

where $\wedge$ is a clockwise closed contour encircling $L$, and $\zeta$ is the coordinate on $\wedge$. According to the Cauchy formula for an infinite region, the contour integral can be calculated; thus general solution (3.29) becomes

$$
\theta(z)=\frac{f}{1-g}+\frac{f}{1-g}(-z+2 i \beta a) X(z)+C X(z) .
$$

The constants $\delta$ and $C$ in (3.34) are determined from the equilibrium conditions of the rigid line. Substituting (3.8) into (2.8), we obtain:

$$
\int_{-a}^{a}\left[\Phi_{1}^{+}(t)+\Omega_{1}^{-}(t)\right] \mathrm{d} t-\int_{-a}^{a}\left[\Phi_{2}^{-}(t)+\Omega_{2}^{+}(t)\right] \mathrm{d} t=0
$$


which can be reduced to an integral along a closed contour $\wedge$ encircling $L$ :

$$
\int_{\Lambda} \theta(z) \mathrm{d} z=0
$$

Similarly, from (2.10), we obtain:

$$
\operatorname{Re} \int_{\Lambda} z \theta(z)=0
$$

Integrals in (3.36) and (3.37) can be evaluated by using the residue theorem, thus we obtain:

$$
\begin{gathered}
C=0 \\
\delta=\operatorname{Im}\left[\frac{\kappa_{1}}{4 \mu_{1}} \Gamma_{1}+\frac{\kappa_{2}}{4 \mu_{2}} \Gamma_{2}-\frac{1}{4 \mu_{1}}\left(\Gamma_{1}+\Gamma_{1}^{\prime}\right)-\frac{1}{4 \mu_{2}}\left(\Gamma_{2}+\Gamma_{2}^{\prime}\right)\right] .
\end{gathered}
$$

Lastly we obtain the solution of the problem:

$$
\theta(z)=\frac{f}{1-g}+\frac{f}{1-g}(-z+2 i \beta a) X(z)
$$

where

$$
f=\frac{\kappa_{1} \mu_{2}+\mu_{1}}{\kappa_{1}} \operatorname{Re}\left[-\left(\frac{\kappa_{1}}{2 \mu_{1}} \Gamma_{1}+\frac{\kappa_{2}}{2 \mu_{2}} \Gamma_{2}\right)+\frac{1}{2 \mu_{1}}\left(\Gamma_{1}+\Gamma_{1}^{\prime}\right)+\frac{1}{2 \mu_{2}}\left(\Gamma_{2}+\Gamma_{2}^{\prime}\right)\right]
$$

In a special case in which the two media are identical, we have

$$
\begin{gathered}
\mu_{1}=\mu_{2}=\mu, \quad \kappa_{1}=\kappa_{2}=\kappa, \quad g=-1, \quad \beta=0 \\
\Gamma_{1}=\Gamma_{2}=\Gamma, \quad \Gamma_{1}^{\prime}=\Gamma_{2}^{\prime}=\Gamma^{\prime} .
\end{gathered}
$$

From (3.2), (3.3), (3.22), (3.25) and (3.40), we obtain

$$
\begin{aligned}
& \Phi(z)=\Gamma+\frac{1}{2} \operatorname{Re}\left(-\Gamma+\frac{\Gamma}{\kappa}+\frac{\Gamma^{\prime}}{\kappa}\right) \cdot\left(1-\frac{z}{\sqrt{z^{2}-a^{2}}}\right) \\
& \Omega(z)=\Gamma+\Gamma^{\prime}+\frac{1}{2} \operatorname{Re}\left(\kappa \Gamma-\Gamma-\Gamma^{\prime}\right) \cdot\left(1-\frac{z}{\sqrt{z^{2}-a^{2}}}\right)
\end{aligned}
$$

which are in agreement with the classical results [1].

\section{STRESS DISTRIBUTION AROUND THE RIGID LINE}

It is of practical importance to investigate the stress distribution around the rigid line, especially at the rigid line end. As an example, we discuss the normal stress $\sigma_{y 1}$ and the shear stress $\tau_{x y 1}$ on the upper surface of the rigid line. From (3.2), (3.3), (3.8), (3.22) and (3.25), it is seen that

$$
\left(\sigma_{y 1}-i \tau_{(x y 1}\right)^{+}=\Gamma_{1}+\Gamma_{1}+\Gamma_{1}^{\prime}+\frac{\mu_{1}}{\kappa_{1} \mu_{2}+\mu_{1}} \theta^{+}(t)-\frac{\kappa_{2} \mu_{1}}{\kappa_{2} \mu_{1}+\mu_{2}} \theta^{-}(t)
$$

Substituting (3.40) into (4.1), we obtain:

$$
\left(\sigma_{y 1}-i \tau_{x y 1}\right)^{+}=A_{1}+i A_{2}+B(-t+2 i \beta a) X^{+}(t) \quad-a<t<a
$$

where

$$
\begin{aligned}
& A_{1}=\operatorname{Re} \Gamma_{1}^{\prime}+\Gamma_{1}+\Gamma_{1}+\frac{f}{1-g}\left(\frac{\mu_{1}}{\kappa_{1} \mu_{2}+\mu_{1}}-\frac{\kappa_{2} \mu_{1}}{\kappa_{2} \mu_{1}+\mu_{2}}\right) \\
& A_{2}=\operatorname{Im} \Gamma_{1}^{\prime} \\
& B=\frac{f}{1-g}\left(\frac{\mu_{1}}{\kappa_{1} \mu_{2}+\mu_{1}}-\frac{\kappa_{2} \mu_{1}}{\kappa_{2} \mu_{1}+\mu_{2}} \cdot \frac{1}{g}\right) .
\end{aligned}
$$


Noting that

$$
X^{+}(t)=\frac{\mathrm{e}^{\pi \beta}}{\sqrt{a^{2}-t^{2}}}\left[\sin \left(\beta \ln \frac{a+t}{a-t}\right)-i \cos \left(\beta \ln \frac{a+t}{a-t}\right)\right]-a<t<a
$$

we obtain:

$$
\begin{gathered}
\sigma_{y \mathrm{l}}^{+}=A_{1}+\frac{B \mathrm{e}^{\pi \beta}}{\sqrt{a^{2}-t^{2}}}\left[-t \sin \left(\beta \ln \frac{a+t}{a-t}\right)+2 \beta a \cos \left(\beta \ln \frac{a+t}{a-t}\right)\right]-a<t<a \\
\tau_{x y \mathrm{l}}^{+}=-A_{2}-\frac{B \mathrm{e}^{n \beta}}{\sqrt{a^{2}-t^{2}}}\left[2 \beta a \sin \left(\beta \ln \frac{a+t}{a-t}\right)+t \cos \left(\beta \ln \frac{a+t}{a-t}\right)\right]-a<t<a .
\end{gathered}
$$

Furthermore, we consider the singular behavior of the stresses at the rigid line end $\left(t \rightarrow a_{-0}\right)$. Let $r=a-t ;$ we then have

$$
\begin{aligned}
\sigma_{y 1}^{+} & =-B \mathrm{e}^{\pi \beta} \sqrt{\frac{a}{2}} r^{-1 / 2}\left[-\sin \left(\beta \ln \frac{2 a}{r}\right)+2 \beta \cos \left(\beta \ln \frac{2 a}{r}\right)\right] r \ll a \\
\tau_{x y 1}^{+} & =-B \mathrm{e}^{x \beta} \sqrt{\frac{a}{2}} r^{-1 / 2}\left[2 \beta \sin \left(\beta \ln \frac{2 a}{r}\right)+\cos \left(\beta \ln \frac{2 a}{r}\right)\right] r \ll a .
\end{aligned}
$$

From (4.9) and (4.10), it is seen that the modulus of the singular behavior of the stresses remains proportional to the inverse square root of the distance from the rigid line end, but the stresses possess a pronounced oscillatory character, as in the case of an interfacial crack tip. One would expect very high stress concentrations at the rigid line end, which may result in cracking.

\section{REFERENCES}

[1] N. I. Muskhelishvili, Some Basic Problems of Mathematical Theory of Elasticity. Noordhof, Leyden (1975).

[2] Q. Q. Li and T. C. T. Ting. Line inclusions in anisotropic elastic solids. J. appl. Mech. 56, 556-563 (1989).

[3] T. H. Hao and Y. C. Wu, Elastic plane problem of collinear periodical rigid lines. Engng Fraciure Mech. 33, 979-981 (1989).

[4] X. Markenscoff and J. Dundurs, Weight functions for rigid line inclusions. Proc. 7th Int. Conf. on Fracture, Houston, TX, Vol. 1, pp. 37-39 (1989).

[5] C. P. Jiang, The plane problem of collinear rigid lines under arbitrary loads. Engng Fracture Mech 39, $299-308$ (1991).

(Received 4 April 1991) 